\title{
Hypertonie: Adhärenz beeinflusst die Prognose
}

\author{
Eine antihypertensive Behandlung schützt Patienten mit Bluthochdruck vor der Entwicklung \\ einer Herzinsuffizienz. Eine Studie unterstreicht die Bedeutung der Therapieadhärenz.
}

_ Die Fall-Kontroll-Studie basiert auf den Daten von 76.017 Patienten im Alter zwischen 40 und 80 Jahren, bei denen eine antihypertensive Behandlung begonnen wurde. 622 Patienten, die wegen Herzinsuffizienz stationär aufgenommen wurden, wurden je bis zu fünf Kontrollen zugeordnet.

Die Adhärenz zur antihypertensiven Therapie wurde definiert über den Anteil der Tage an der Gesamttherapiedauer, an denen die Medikamente eingenommen wurden. Die vierstufige Skala ging von sehr niedrig (Einnahme an weniger als 25\% der Tage) über niedrig und mittel bis hoch (mehr als 75\% der Tage).
Das Risiko einer stationär behandlungspflichtigen Herzinsuffizienz war bei mittlerer und hoher Adhärenz signifikant geringer als bei sehr niedriger Adhärenz. Das Lebensalter der Patienten spielte offenbar für diesen Zusammenhang keine Rolle: Die Altersgruppe von 40-70 Jahren und die von 71-80 Jahren hatten vergleichbare Ergebnisse.

Unterschiede gab es zwischen den Medikamentengruppen: Die Adhärenz zu ACE-Hemmern, $\mathrm{AT}_{1}$-Rezeptor-Blockern und Diuretika hatte einen signifikanten Einfluss auf das Herzinsuffizienzrisiko, die Adhärenz zu Kalziumantagonisten hingegen nicht.
- Corrao G, Rea F, Ghirardi A et al. Adherence With Antihypertensive Drug Therapy and the Risk of Heart Failure in Clinical Practice. Hypertension. 2015;66:742-9

\section{KOMMENTAR}

Die Studie ist insofern interessant, als sie auch hinsichtlich des Herzinsuffizienzrisikos zeigt, dass die Einnahmetreue keine theoretische Größe auf dem Papier ist, sondern die Prognose speziell hinsichtlich der Entwicklung kardialer Endorganschäden wesentlich beeinflusst. Die Ergebnisse sind somit für die Praxis ein besonderer Ansporn, die von solchen Endorganschäden besonders bedrohten Patienten zur regelmäßigen Einnahme ihrer Antihypertensiva zu motivieren.

Prof. Dr. med. W. Zidek

\section{Dieser Pleuraerguss ist pechschwarz eingefärbt}

Bei einem 56-jährigen Mann mit Prostatakarzinom wurde ein ausgedehnter rechtsseitiger Pleuraerguss festgestellt (Abb. A). Trotz der GröBe des Ergusses hatte der Mann nur eine geringe Atemnot und wurde ursprünglich wegen Harninkontinenz stationär aufgenommen. Bei der Pleurapunktion entleerte sich schwarze Flüssigkeit (Abb. B). Es handelte sich um ein Ex-
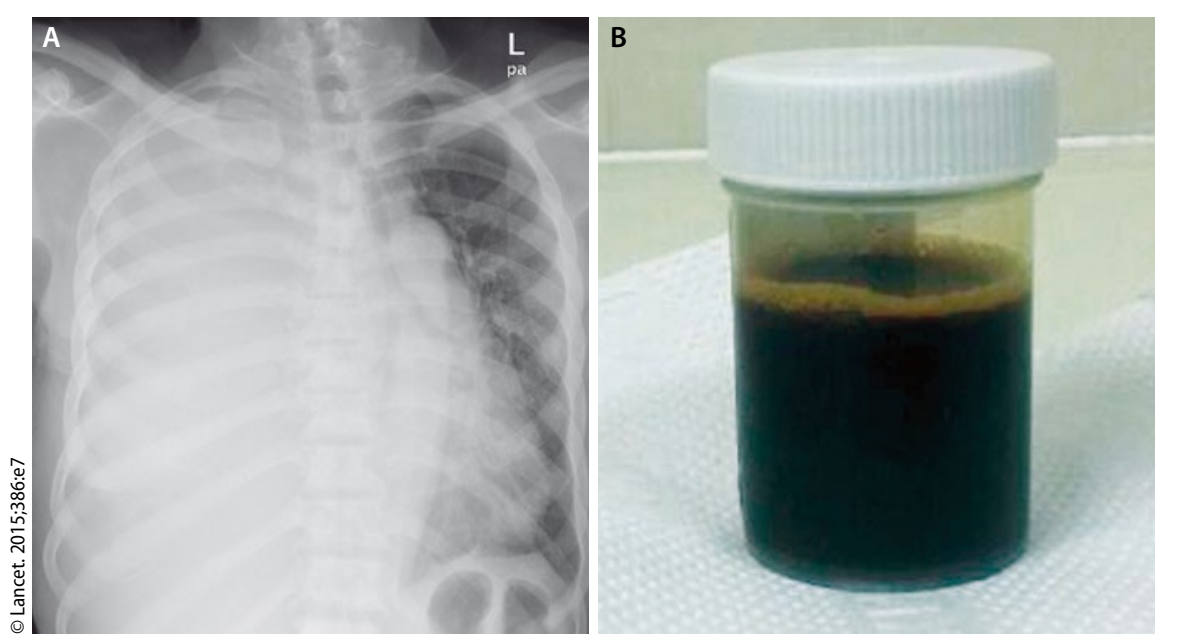

Ausgedehnter rechtsseitiger Pleuraerguss im Röntgenthorax (A), schwarze Flüssigkeit

aus der Pleurahöhle (B).

sudat mit hohen Konzentrationen von Eiweiß $(85 \mathrm{~g} / \mathrm{l})$, Lactatdehydrogenase und Amylase. Über 24 Stunden hinweg wurden 3 I Pleuraerguss abdrainiert. Während die Bronchoskopie unauffällig ausfiel, zeigte sich in der Thorakoskopie eine Verdickung der viszeralen und der parietalen Pleura mit zahlreichen Knötchen. Zytologisch fand man im Pleuraerguss maligne, drüsenartig angeordnete Zellen, daneben Mesothelzellen und Entzündungszellen in einer hämorrhagischen Flüssigkeit. Immunhistochemisch bestanden im Erguss Hinweise auf ein Adenokarzinom der Lunge. Die Pleurabiopsie bestätigte diese Diagnose. Der Patient erhielt zur Behandlung Zytostatika.

Schwarzgefärbte Pleuraergüsse sind extrem selten und wurden bisher nur bei Patienten mit fungaler Pleuritis (Aspergillus niger oder Rhizopus oryzae), metastasierenden Melanomen, Adenokarzinomen der Lunge, pankreatikopleuralen Fisteln oder Ösophagusperforationen bei der Applikation von Aktivkohle sowie bei Kokainmissbrauch berichtet. Die Schwarzverfärbung der Pleuraflüssigkeit im vorliegenden Fall ist wahrscheinlich durch eine Hämolyse und die Anwesenheit von hämosiderinbeladenen Makrophagen nach einer massiven Blutung in die Pleurahöhle bedingt - dies aber wohl schon lange Zeit vor der Vorstellung des Patienten. Erhöhte Konzentrationen von Amylase in der Pleuraflüssigkeit sind ebenfalls typisch für ein Adenokarzinom.

Prof. Dr. med. H. S. FüeßI

- Jayakrishnan B et al. (drjayakrish@hotmail.com). Black pleural effusion. Lancet. 2015;386:e7 